\title{
Where's the Excitement in Psychostimulant Sensitization?
}

Repeated psychostimulant exposure produces sensitized behavioral responses that persist into withdrawal and are thought to model increased drug craving in psychostimulant abusers (Post and Rose 1976; Robinson and Berridge 1993; Stewart 1983). A potential mechanism for inducing behavioral sensitization could involve a transient increase in AMPA glutamate receptor-mediated excitability in ventral tegmental area (VTA) dopamine neurons (White et al. 1995; Zhang et al. 1997). Previously, this hypersensitivity was thought to involve transient increases in AMPA (and NMDA) receptor subunits after multiple drug or stress-related treatment regimens known to induce behavioral sensitization (Churchill et al., 1999; Fitzgerald et al. 1996).

However, a study by Lu, Monteggia, and Wolf published in this issue of Neuropsychopharmacology directly challenges the idea that psychostimulant-induced hypersensitivity in VTA dopamine neurons involves an increase in the actual amount of glutamate receptors. In this study, multiple treatment regimens with amphetamine and cocaine, known to induce behavioral sensitization, and identical to protocols used in previous studies, all failed to increase AMPA subunits in the VTA and neighboring substantia nigra. Such discrepancies in the drug abuse literature usually are attributed to differences in dose, chronicity, withdrawal time, self- versus passive drug administration, or other methodological considerations.

The study by Lu et al. $(1999,2001)$ used quantitative immunohistochemical and reverse transcriptase-polymerase chain reaction methods to measure GluR1-4 levels throughout the rostral-caudal extent of the VTA and substantia nigra, whereas increases in AMPA subunits previously were detected in immunoblots of VTA tissue. After a series of experiments to verify epitope and sequence specificity of the GluR1 antibody and RNA probe, respectively, the authors conducted an exhaustive and thorough search for the precise anatomical site in VTA where the elusive upregualtion in GluR1 subunits was thought to occur. They found no evidence for upregulation of AMPA subunits at the mRNA or protein level in any region of VTA and substantia nigra, whether at 16 and $24 \mathrm{~h}$ or 3 and 14 days after multiple chronic treatment regimens with amphetamine and cocaine. Other studies have also failed to find evidence for upregulation in mRNAs for AMPA GluR1 or NMDA NR1 subunits, suggesting that reported increases in GluR1 and NR1 protein may not involve changes in GluR1 gene expression (Bardo et al., 2001; Ghasemzadeh et al., 1999). However, the present study also failed to find increases in GluR1 protein. One potential caveat to the immunohistochemical method used by Lu et al. is that GluR1 antibodies could recognize other endogenous proteins containing GluR1-like epitopes that dilute changes in GluR1 subunits themselves in native tissue slices; this problem was circumvented in previous studies in which GluR subunits were isolated by standard SDS PAGE techniques before immunolabeling. However, the authors note that their immunohistochemical method is capable of detecting changes as small as $15 \%$ in other brain regions. What may be apparent from this and other studies is that relatively similar chronic drug treatments produce markedly different effects on AMPA subunits in VTA across several laboratories (see Table 1).

In the present study, Lu et al. conclude that transient increases in GluR1 protein cannot account for transient increases in AMPA-mediated electrophysiological responses associated with the induction of behavioral sensitization, and they suggest phosphorylation or membrane trafficking of AMPA receptors as alternative hypotheses. There is considerable evidence for phosphorylation and membrane trafficking as important events in AMPA receptor plasticity in long-term potentiation (LTP) and long-term depression (LTD) (Carroll et al. 2001; Scannevin and Huganir 2000). Indeed, a recent 


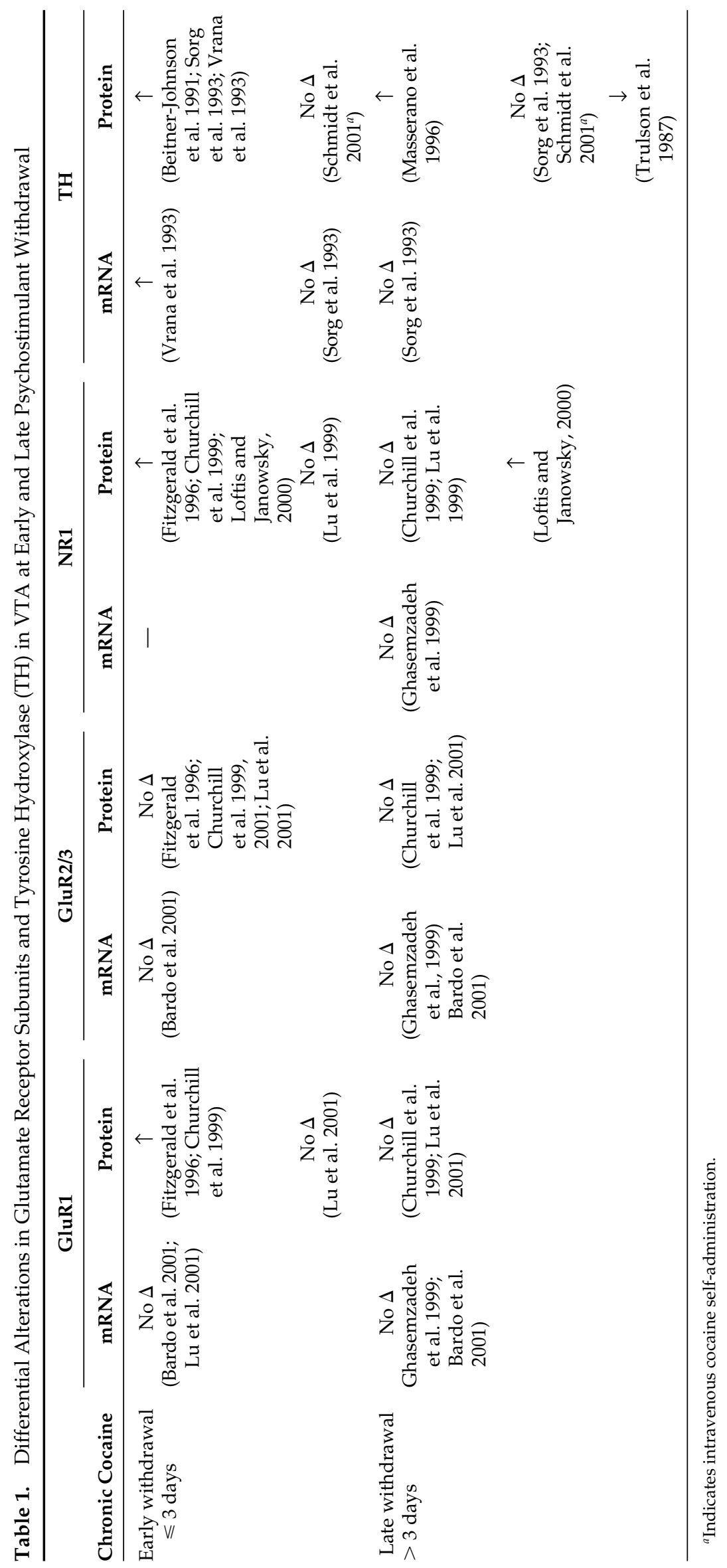


study found that a single injection of cocaine produces both behavioral sensitization and LTP in VTA dopamine neurons independent of detectable AMPA receptor upregulation (Ungless et al. 2001), thereby implicating other possible trafficking and/or phosphorylation alterations. However, others have found that "synaptic" concentrations of AMPA receptors actually do increase after LTP in the hippocampus, and such subanatomical changes may not be detected in tissue slices or crude tissue homogenates (Heynen et al. 2001).

It is clear from these studies that drug-induced neuroadaptations in the VTA differ widely across research laboratories, even when drug regimens and withdrawal times are taken into account. Several studies on psychostimulant regulation of glutamate receptor subunits in the VTA are listed in Table 1, along with regulation of VTA tyrosine hydroxylase levels, which also differ among studies and psychostimulant treatment regimens. It is important to consider that several methodological variables differ between these studies, such as rat strains and vendors and whether animals received drug treatments in their home cages or in specific drugassociated test chambers. Drug-induced neuroplasticity is profoundly altered by certain contextual or other experience-related factors (e.g., Bell et al. 2000; Schmidt et al. 2001), and the potential influence of these factors should not be underestimated.

Results from behavioral studies are sometimes encumbered by the fact that certain effects clearly and easily obtained in one laboratory are difficult to repeat in another. Even when animal strains are held constant and procedures are carefully controlled, different, and even opposite, results can be found (Crabbe et al. 1999). The findings of Giorgetti and colleagues (Gioergetti et al. 2001), when taken in view of previous studies, highlight the fact that neurobiological studies can suffer a similar dilemma. However, in scientific research, these discrepancies serve well to remind us that a single study cannot, and should not, ever be considered definitive. Scientific progress benefits from both convergent and divergent lines of evidence, and discrepant findings such as these ultimately help to illuminate intricate facets of neural and behavioral plasticity that may have important implications for drug addiction research and treatment.

David W. Self, Ph.D. Department of Psychiatry UT Southwestern Medical Center Dallas, TX

\section{REFERENCES}

Bardo MT, Robinet PM, Mattingly BA, Marguilles JE (2001): Effect of 6-hydroxydopamine or repeated amphetamine treatment on mesencephalic mRNA levels for AMPA glutamate receptor subunits in the rat. Neurosci Lett 302:133-136

Beitner-Johnson D, Guitart X, Nestler EJ (1991): Dopaminergic brain reward regions of Lew's and Fischerrats display different levels of tyrosine hydroxylase and other morphine and cocaine-regulated phosphoproteins. Brain Res 561: 147-150

Bell K, Duffy P, Kalivas PW (2000): Context-specific enhancement of glutamate transmission by cocaine. Neuropsychopharmacology 23:335-344

Carroll RC, Beattie EC, von Zastrow M, Malenka RC (2001): Role of AMPA receptor endocytosis in synaptic plasticity. Nature Rev Neurosci 2:315-324

Churchill L, Swanson CJ, Urbina M, Kalivas PW (1999): Repeated cocaine alters glutamate receptor subunit levels in the nucleus accumbens and ventral tegmental area of rats that develop behavioral sensitization. J Neurochem 72:2397-2403

Crabbe JC, Wahlsten D, Dudek BC (1999): Genetics of mouse behavior: Interactions with laboratory environment. Science 284:1670-1672

Fitzgerald LW, Ortiz J, Hamedani AG, Nestler EJ (1996): Regulation of glutamate receptor subunit expression by drugs of abuse and stress: Common adaptations among cross-sensitizing agents. J Neurosci 16:274-282

Ghasemzadeh MB, Nelson LC, Lu X-Y, Kalivas PW (1999): Neuroadaptations in ionotropic and metabotropic glutamate receptor mRNA produced by cocaine treatment. J Neurochem 72:157-165

Giorgetti M, Hotsenpiller G, Ward P, Tepper T, Wolf ME (2001): Amphetamine-induced plasticity of AMPA receptors in the ventral tegmental area: Effects on extracellular levels of dopamine and glutamine in freely moving rats. J Neurosci 21:6362-6369

Heynen AJ, Quinlan EM, Bae DC, Bear MF (2001): Bidirectional, activity-dependent regulation of glutamate receptors in the adult hippocampus in vivo. Neuron 28:527-536

Loftis JM, Janowsky A (2000): Regulation of NMDA receptor subunits and nitric oxide synthase expression during cocaine withdrawal. J Neurochem 75:2040-2050

Lu W, Monteggia LM, Wolf ME (1999): Withdrawal from repeated amphetamine administration reduces NMDAR1 expression in the rat substantia nigra, nucleus accumbens and medial prefrontal cortex. Eur J Neurosci 11:3167-3177

Lu W, Monteggia LM, Wolf ME (2001) Repeated administration of amphetamine and cocaine does not alter AMPA receptor subunit expression in the rat midbrain. Neuropsychopharmacology 26:1-13.

Masserano JM, Baker I, Natsukari N, Wyatt RJ (1996): Chronic cocaine administration increases tyrosine hydroxylase activity in the ventral tegmental area through glutaminergic- and dopaminergic D2-receptor mechanisms. Neurosci Lett 217:73-76

Post R, Rose H (1976): Increasing effects of repetitive cocaine administration in rats. Nature 260:731-734

Robinson TE, Berridge KC (1993): The neural basis of drug craving: An incentive-sensitization theory of addiction. Brain Res Rev 18:247-291

Scannevin RH, Huganir RL (2000): Postsynaptic organiza- 
tion and regulation of excitatory synapses. Nature Rev Neurosci 1:133-141

Schmidt EF, Sutton MA, Schad CA, Karanian DA, Brodkin ES, Self DW (2001): Extinction training regulates tyrosine hydroxylase during withdrawal from cocaine selfadministration. J Neurosci 21:RC137

Sorg BA, Chen S-Y, Kalivas PW (1993): Time course of tyrosine hydroxylase expression after behavioral sensitization to cocaine. J Phamacol Exp Ther 266:424-430

Stewart J (1983): Conditioned and unconditioned drug effects in relapse to opiate and stimulant drug selfadminstration. Prog Neuro-Psychopharmacol Biol Psychiatry 7:591-597

Trulson ME, Cannon MS, Faegg TS, Raese JD (1987): Tyrosine hydroxylase immunochemistry and quantitative light microscopic studies of the mesolimbic dopamine system in rat brain: effects of chronic methamphetamine administration. Brain Res Bull 18:269-277
Ungless MA, Whistler JL, Malenka RC, Bonci A (2001): Single cocaine exposure in vivo induces long-term potentiation in dopamine neurons. Nature 411:583-587

Vrana SL, Vrana KE, Koves TR, Smith JE, Dworkin SI (1993): Chronic cocaine administration increases CNS tyrosine hydroxylase enzyme activity and mRNA levels and tryptophan hydroxylase enzyme activity levels. J Neurochem 61:2262-2268

White FJ, Hu XT, Zhang XF, Wolf ME (1995): Repeated administration of cocaine or amphetamine alters neuronal responses to glutamate in the mesoaccumbens dopamine system. J Pharmacol Exp Ther 273:445-454

Zhang X-F, Hu X-T, White FJ, Wolf ME (1997): Increased responsiveness of ventral tegmental area dopamine neurons to glutamate after repeated administration of cocaine or amphetamine is transient and selectively involves AMPA receptors. J Pharmacol Exp Ther 281: 699-706 